\title{
Cardiorespiratory Coupling Functions, Synchronization and Ageing
}

\author{
Tomislav Stankovski, Peter V. E. McClintock, and Aneta Stefanovska*
}

\begin{abstract}
We discuss the advent of coupling functions as a new dimension in the analysis of cardiorespiratory interactions. By decomposing the separate coupling components in a phase oscillator model, we infer the cardiorespiratory coupling functions with dynamical Bayesian inference for time-evolving and noisy coupled systems. The inferred coupling functions are themselves time-varying processes. On a longer timescale, application to ageing shows that the coupling functions and the direct influence of respiration decrease as age increases. We find that the coupling functions can give rise to synchronization transitions, and that there are no significant changes in synchronization with age.
\end{abstract}

\section{INTRODUCTION}

The cardiorespiratory interactions have been described successfully through the investigation of oscillatory timeseries acquired through noninvasive measurements. Two main groups of analyses for assessing the interactions of cardiorespiratory oscillations recur in the literature: detection of phase-locking or synchronization [1], [2], [3]; and detection of coupling strength or directionality [4], [5], [6].

Recently, a new dimension of the cardiorespiratory interactions - the coupling function, became available [7], [8], [9]. Coupling functions prescribe the physical rule specifying how the cardiorespiratory inter-oscillator interactions occur [10], [7]. In this way, the coupling functions reveal information not only about the coupling strength, but also about the functional form of the coupling. They determine the possibility of qualitative transitions between the oscillations e.g. routes into, and out of, phase synchronization. Their decomposition can describe the separate functional contributions from either the heart or respiration.

The cardiorespiratory coupling functions are inherently time-evolving processes [7]. On a longer timescale, their analysis can be used to study the evolution of human ageing [8]. By investigating the separate direct contributions or phase resetting curves, it was found that the cardiorespiratory coupling is attributable mostly to respiratory sinus arrythmia (RSA) [8], [9]. The work has also inspired the development of an improved means of secure communications [11].

\section{MODEL AND INFERENCE OF CARDIORESPIRATORY INTERACTIONS}

We model the cardiorespiratory system as a pair of noisy coupled phase oscillators with phases $\phi_{h}(t)$ and $\phi_{r}(t)$. The influence of the respiration on the heart is:

$$
\begin{aligned}
\dot{\phi}_{h}(t) & =\omega_{h}(t)+q_{h}\left(\phi_{h}, \phi_{r}, t\right)+\xi_{h}(t) \equiv \omega_{h}(t)+ \\
& +\quad s_{h}\left(\phi_{h}, t\right)+d_{h}\left(\phi_{r}, t\right)+i_{h}\left(\phi_{h}, \phi_{r}, t\right)+\xi_{h}(t),
\end{aligned}
$$

Research supported by the Engineering and Physical Sciences Research Council (UK) (grant no. EP/100999X1).

T. Stankovski, P. V. E. McClintock, and A. Stefanovska are with the Department of Physics, Lancaster University, Lancaster, LA1 4YB, UK. (*Corresponding author's e-mail: aneta@lancaster.ac.uk). where the $h$ subscript indicates the heart and $r$ the respiration, $\omega_{h, r}$ denote their natural frequencies, and $\xi_{h, r}$ is assumed to be white Gaussian noise. The coupling functions $q_{h}\left(\phi_{h}, \phi_{r}, t\right)$ are separated into three parts: a self-interaction $s_{h, r}$ which accelerates/slows phase growth depending on the current phase of the oscillator considered; a direct interaction $d_{h, r}$ which accelerates/slows phase growth depending on the current phase of the other oscillator; and an indirect part $i_{h, r}$ which depends on both phases. The instantaneous phases $\phi_{h}(t)$ and $\phi_{r}(t)$ were estimated using the synchrosqueezed wavelet transform [12].

In view of the periodic nature of the phase dynamics we represent the functions of the phase model with Fourier series, and employ dynamical Bayesian inference [7], [13] to evaluate the model and its parameters from the phase time-series. The method is specially designed to allow for inference of time-varying parameters. This approach yields the effective coupling, providing information about causality and the form of the coupling functions. The application of dynamical inference allows one to infer the functional mechanisms, which is a significant advantage over statistical methods such as Granger causality and transfer entropy, where one can infer only statistical effects [14].

\section{CARDIORESPIRATORY COUPLING FUNCTIONS AND THEIR TIME-EVOLUTION}

Cardiorespiratory coupling functions were introduced (arguably for the first time) by being evaluated for subjects whose paced respiration was being ramped down, i.e. with decreasing frequency (see Fig. 1 of [7], and [15] for details). It was found that the cardiorespiratory coupling functions can themselves be time-varying processes. Temporally-resolved coupling functions can change very significantly with time, so that, only by comparing consecutive estimations can one follow the evolution of their form (Fig. 1).

Qualitatively, the respiration-to-heart coupling function, e.g. as shown in Fig. 1(b), has a sine-like waveform along the respiration $\phi_{1}$-axis, while being mostly constant along the
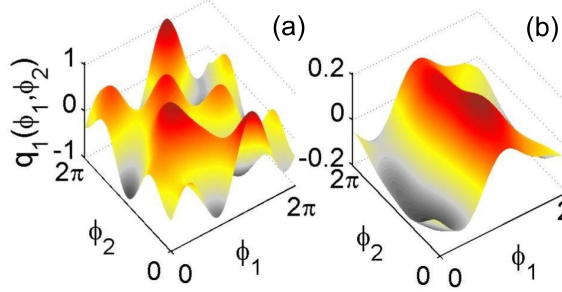

(b)

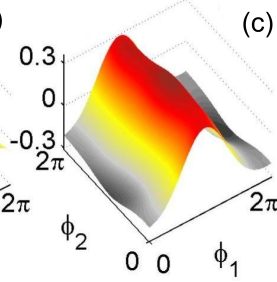

Fig. 1. Cardiorespiratory coupling function showing the respiration-to-heart influence from one subject. (a)-(c) are evaluated for different time windows (50s long): (b) and (c) are for consecutive windows, much later than (a). The index 1 is for respiration and index 2 for the heart phase. 


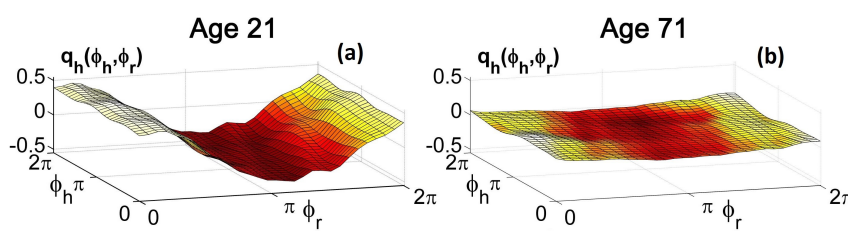

Fig. 2. Cardiorespiratory coupling function showing the respiration-to-heart influence from (a) young and (b) older subject.

heart-axis. This strongly implies that much of the cardiorespiratory coupling is attributable to the direct contribution from the respiratory oscillation.

\section{CARDIORESPIRATORY COUPLING FUNCTIONS AND AGEING}

The method can also be used [8] to reveal the evolution of cardiorespiratory interactions with age [16]. The direct coupling contribution $d_{r}$ in the $\phi_{h}$ dynamics, identified as RSA modulation, was found to decrease significantly with age. Fig. 2(a) and (b) show the time-averaged coupling functions $q_{h, r}$ typical of a younger and an older subject, respecively. Decrease of the direct influence from respiration (or RSA amplitude) with age is clearly evident. It can also be concluded that the main stable contribution to $q_{h}$, surviving after time-averaging, remains RSA irrespective of age. The respiratory coupling $q_{r}$ was found to be quite irregular and not age-dependent.

\section{CARDIORESPIRATORY SYNCHRONIZATION}

Having inferred the parameters of the phase model we can determine whether such phase oscillators undergo synchronization: in this way one detects intrinsic synchronization i.e. the synchronized state resulting from coupling. By decomposing the noise effects with Bayesian inference, one can also detect the nature of phase-slips, establishing whether they are noise- or coupling-induced [7], [13].

For the (ramped) time-varying respiration frequency, the system undergoes transitions into and out of synchrony, and between different synchronization ratios [7]. Interestingly, there is no significant change in the cardiorespiratory synchronization state between different ageing groups [8]. The commonest cardiorespiratory synchronization ratio was $4: 1$.

\section{CONCLUSIONS}

We have presented coupling function analysis as an important new means of quantifying the cardiorespiratory interactions. The functional form of the coupling from the respiration to the heart indicated that the cardiorespiratory coupling is mostly on account of the direct $d_{h}$, and not the self $s_{h}$ or indirect $i_{h}$ coupling components. The direct influence has been identified as the RSA physiological modulation [8]. The same conclusion was reached by observation of the cardiorespiratory phase-resetting curves [9].

We have shown that the form of the cardiorespiratory coupling can be time-evolving. These temporal states, which could lead to synchronization transitions, can be reduced out in the overall time-averaged form. Another observation was that, even though the coupling functions were timevarying, the existence of the interaction was stable and persisting - perhaps reflecting the chronotaxic nature of the cardiorespiratory interactions [17], [18].

The application of the method to ageing has led to some important conclusions [8]. They imply inter alia that the analysis of coupling functions also promises important applications to other physiological interactions.

\section{REFERENCES}

[1] A. Stefanovska, H. Haken, P. V. E. McClintock, M. Hožič, F. Bajrović, and S. Ribarič, "Reversible transitions between synchronization states of the cardiorespiratory system," Phys. Rev. Lett., vol. 85, no. 22, pp. 4831-4834, 2000.

[2] C. Schäfer, M. G. Rosenblum, J. Kurths, and H. H. Abel, "Heartbeat synchronised with ventilation," Nature, vol. 392, no. 6673, pp. 239240, 1998.

[3] D. A. Kenwright, A. Bahraminasab, A. Stefanovska, and P. V. E. McClintock, "The effect of low-frequency oscillations on cardiorespiratory synchronization," Eur. Phys. J. B., vol. 65, no. 3, pp. 425433, 2008.

[4] M. Paluš and A. Stefanovska, "Direction of coupling from phases of interacting oscillators: An information-theoretic approach," Phys. Rev. $E$, vol. 67, p. 055201(R), 2003.

[5] M. G. Rosenblum and A. S. Pikovsky, "Detecting direction of coupling in interacting oscillators," Phys. Rev. E., vol. 64, no. 4, p. 045202, 2001.

[6] L. Faes, A. Porta, R. Cucino, S. Cerutti, R. Antolini, and G. Nollo, "Causal transfer function analysis to describe closed loop interactions between cardiovascular and cardiorespiratory variability signals," $\mathrm{Bi}$ ological cybernetics, vol. 90, no. 6, pp. 390-399, 2004.

[7] T. Stankovski, A. Duggento, P. V. E. McClintock, and A. Stefanovska, "Inference of time-evolving coupled dynamical systems in the presence of noise," Phys. Rev. Lett., vol. 109, p. 024101, 2012.

[8] D. Iatsenko, A. Bernjak, T. Stankovski, Y. Shiogai, P. J. OwenLynch, P. B. M. Clarkson, P. V. E. McClintock, and A. Stefanovska, "Evolution of cardio-respiratory interactions with age," Phil. Trans. R. Soc. Lond. A, vol. 371, no. 1997, p. 20110622, 2013.

[9] B. Kralemann, M. Frühwirth, A. Pikovsky, M. Rosenblum, T. Kenner, J. Schaefer, and M. Moser, "In vivo cardiac phase response curve elucidates human respiratory heart rate variability," Nat. Commun., vol. 4, 2013.

[10] B. Kralemann, L. Cimponeriu, M. Rosenblum, A. Pikovsky, and R. Mrowka, "Phase dynamics of coupled oscillators reconstructed from data," Phys. Rev. E, vol. 77, no. 6, Part 2, p. 066205, 2008.

[11] T. Stankovski, P. V. E. McClintock, and A. Stefanovska, "Coupling functions enable secure communications," Phys. Rev. X, 2014.

[12] I. Daubechies, J. Lu, and H. Wu, "Synchrosqueezed wavelet transforms: An empirical mode decomposition-like tool," Appl. and Comput. Harmon. Anal., vol. 30, no. 2, pp. 243-261, 2011.

[13] A. Duggento, T. Stankovski, P. V. E. McClintock, and A. Stefanovska, "Dynamical Bayesian inference of time-evolving interactions: From a pair of coupled oscillators to networks of oscillators," Phys. Rev. E, vol. 86, p. 061126, 2012.

[14] K. Friston, R. Moran, and A. K. Seth, "Analysing connectivity with granger causality and dynamic causal modelling," Current opinion in neurobiology, 2012.

[15] T. Stankovski, W. H. Cooke, L. Rudas, A. Stefanovska, and D. L. Eckberg, "Time-frequency methods and voluntary ramped-frequency breathing: a powerful combination for exploration of human neurophysiological mechanisms," J. Appl. Physiol., vol. 115, no. 12, pp. 1806-1821, 2013

[16] Y. Shiogai, A. Stefanovska, and P. V. E. McClintock, "Nonlinear dynamics of cardiovascular ageing," Phys. Rep., vol. 488, pp. 51-110, 2010.

[17] Y. F. Suprunenko, P. T. Clemson, and A. Stefanovska, "Chronotaxic systems: A new class of self-sustained nonautonomous oscillators," Phys. Rev. Lett., vol. 111, no. 2, p. 024101, 2013.

[18] Y. F. Suprunenko, P. T. Clemson, and A. Stefanovska, "Chronotaxic systems with separable amplitude and phase dynamics," Phys. Rev. E, vol. 89, no. 1, p. 012922, 2014. 